\title{
Incidencia de competencias parentales en el desarrollo de habilidades sociales en hijos únicos
}

\section{Incidence of parental competences in the development of social skills}

\author{
in kids from single children families
}

\author{
Magaly Pacheco Marimon ${ }^{1}$ y Gloria Yanet Osorno Álvarez ${ }^{2}$
}

${ }^{1}$ Socióloga. Magister en Ciencias Sociales y Humanas. Master en Ciencias de la Educación. Docente de la Corporación Universitaria Minuto de Dios. Docente de la Universidad de Antioquia, Colombia. Docente del Politécnico Jaime Isaza Cadavid. Coordinadora en el

Semillero de Psicología "Familia, Sociedad y Salud Mental".

E-mail: magaly.pacheco@uniminuto.edu

${ }^{2}$ Psicóloga. Tecnóloga en costos y auditoría. E-mail: gloriaosorno04@gmail.com

El presente artículo da cuenta de la investigación "Incidencia de las pautas de crianza moderna en el desarrollo de las habilidades sociales, de niños y niñas de 3 a 5 años, hijos únicos, en el Centro Infantil Castillo de Sueños, Municipio de Apartadó". La entidad financiadora de la misma fue la Corporación Universitaria Minuto de Dios.

Código del proyecto: 520-IN-1-16-018.

\section{Antioquia, Colombia}

\section{Resumen}

Múltiples investigaciones han demostrado que los estilos de crianza influyen en el desarrollo socioemocional durante la infancia, en la cual se considera a la familia como el primer ente socializador. El objetivo de esta investigación fue analizar la incidencia de las competencias parentales en el desarrollo de las habilidades sociales de hijos únicos, con edades entre tres y cinco años, teniendo en cuenta cuatro áreas: vinculares, formativas, protectoras y reflexivas. Con respecto a las habilidades sociales se analizaron seis actitudes comportamentales: habilidades sociales básicas; hacer amigos; conversacionales; sentimientos y emociones; capacidad de afrontar y resolver problemas, y la forma de relacionarse con los adultos. Es una investigación cuantitativa, realizada con un método correlacional donde la muestra correspondió a 36 niños y 61 padres de familia. Se utilizaron las siguientes pruebas: Escala de parentalidad positiva y Escala de habilidades sociales.

Los hallazgos de esta investigación no coinciden con lo que teóricamente se podría esperar; resulta paradójico que los niños que tienen habilidades sociales medias posean padres con competencias parentales óptimas, mientras que niños con habilidades sociales altas, presentan padres de familia cuyas competencias parentales que se ubican en cualquiera de los tres rangos (en riesgo, en monitoreo y óptimas). Otro hallazgo se relaciona con los niños que presentaron habilidades sociales altas en las seis áreas; a su vez, sus padres registraron competencias parentales protectoras en zona de monitoreo, lo que conlleva a pensar, que existen otros factores diferentes de las competencias parentales que 
pueden incidir en el desarrollo de las habilidades sociales de los menores.

Palabras clave: habilidades sociales, competencia parental, pautas de crianza, familia, hijos únicos.

\section{Abstract}

Multiple researches have shown that parenting styles influence socio-emotional development during childhood, in which the family is considered the first socializing entity. The objective of this research was to analyze the incidence of parental competences in the development of the social skills of single children aged between three and five years, taking into account four areas: linkage, formative, protective and reflexive. Regarding social skills, six behavioral attitudes were analyzed: basic social skills; making new friends; conversational; feelings and emotions; ability to face and solve problems; and way of relating to adults. It is a quantitative research, correlational method where the sample corresponded to 36 children and 61 parents. Two tests were applied: Positive Parentally Scale and Social Skills Scale. Pearson Chi-square was used to analyze the information, using Statistica software. The Pearson Chi-square test is a non-parametric test, which aims to determine the existence or not of a significant relationship between two variables.

The Parental Competence scale is a very simple questionnaire that applies to the parents or caregivers of a child or adolescent, whose objective is to determine the degree of development of parental competencies that adults use to establish a relationship with the child in care, either as a parent or as a caregiver. The scale groups these competences into four areas: Link, training, protection and reflection. This scale defines three zones to determine the level of competence of the parents: the risk zone, where the parents whose scores are below the reference sample are located; subsequently, the monitoring area is located, where the parents who present scores with percentiles between 30 and 40 are located according to the reference sample. Finally, the optimal zone is defined, where the parents with high scores are located.

The social skills scale of Lacunza is a questionnaire that must be applied by parents or caregivers to children between 3 and 5 years old, which measure observable behaviors, which account for the child's social performance during the last three months. This questionnaire is based on other instruments that measure social skills, but makes an adaptation according to the developmental stages of development in preschool children, perfecting three questionnaires according to age: for 3 years the scale presents a composite questionnaire for 12 items; At the age of 4 years, the evaluation is carried out through the development of 12 questions, while for the age of 5 the questionnaire is made up of a total of 15 items.

The findings of this research do not match what theoretically would be expected. It is paradoxical that children who have medium social skills have parents with optimal parental skills, while children with high social skills, have parents whose parental skills are located in any of the three ranges (at risk, monitoring and optimal). Another finding is related to children who presented high social skills in the six areas, in turn, their parents registered protective parental competencies in the monitoring area, which leads us to think that there are other factors that can affect in the development of the social skills of minors.

Keywords: social skills, parental competence, parenting guidelines, family, single children.

\section{Introducción}

La familia es el primer contexto social para la transmisión de normas, valores y modelos de comportamiento Permite que el niño aprenda formas de socialización a partir de la interiorización de elementos básicos de su cultura (Muñoz, 2009). Se considera que la familia es el primer agente formador, tiene 
influencia directa sobre el desarrollo socioafectivo, además de la adquisición de normas, valores y habilidades. Es en el contexto familiar en el cual se dan las primeras pautas que permiten el desarrollo de las conductas que influirán en la relación e interacción social del niño fuera de su núcleo familiar. En la misma línea, diferentes investigadores ratifican la importancia de la familia y adecuadas relaciones parentales en el desarrollo social, afectivo, cognitivo y emocional de cualquier sujeto durante su vida, en especial durante la infancia (Bronfenbrenner y Evans, 2000; Muñoz Silva, 2005, Vargas Rubilar, Lemos y Richaud, 2017).

The World Family Map del Social Trends Institute (2014) afirma que la familia es una institución fundamental para la educación infantil en la cual se fomenta el desarrollo positivo en los niños. Además, aduce que los índices de natalidad en Centro y Sur América han disminuido de manera significativa durante las últimas décadas y se ha incrementado el crecimiento de hogares con hijos únicos. Frente al fenómeno del hijo único existen diferentes posiciones. Por una parte, están quienes consideran que ellos pueden desarrollar mayores competencias emocionales y sociales que en una familia con más de un hijo. Otros sostienen que son personas emocionalmente inmaduras con dificultad para afrontar la frustración. Pero más allá de estas impresiones subjetivas, la familia es un factor determinante en el desarrollo de la personalidad. Teniendo en cuenta lo anterior, es importante analizar cómo las competencias parentales o pautas de crianza modernas inciden en la adquisición de las habilidades sociales en los niños, específicamente en los hijos únicos.

\section{Competencias parentales}

La crianza hace referencia a la educación y formación que reciben los niños de sus padres y cuidadores. En este sentido, la crianza implica tres procesos psicosociales: pautas, prácticas y creencias acerca de la crianza. Por un lado, las pautas se transmiten de generación en generación y se relacionan con la normatividad que siguen los padres frente al comportamiento de los hijos y son portadoras de significaciones sociales. Las prácticas de crianza se asocian con las condiciones propias de cada familia, en la cual los padres son los principales encargados de educar a sus hijos de acuerdo con sus propias creencias y vivencias, que generalmente están relacionadas con la crianza que recibieron de sus padres. Por último, las creencias de crianza están relacionadas con los conocimientos que tienen los padres sobre cómo se deben criar los hijos (Izzedin y Pachajoa, 2009). Por otro lado, Barudy y Dantagnan (2005) exponen que las relaciones parentales sanas provista de buen trato, afecto, comunicación asertiva procura a los infantes recursos que le permiten enfrentar los desafíos del crecimiento, desarrollar actitud de resiliencia frente a las adversidades de la vida y resolver problemas en el momento indicado.

Las condiciones psicosociales inciden en el desarrollo de las competencias parentales. Así lo estiman (Rodrigo López, Martín Quintana, Cabrera Casimiro y Máiquez Chaves, 2009) cuando afirman que la monoparentalidad, el nivel educativo de los padres, las condiciones económicas, el contexto de vida y otros factores convierten el rol de ser padres en una actividad muy compleja. Si los padres contribuyen a potenciar la autoestima de sus hijos, se puede aminorar los efectos negativos de contextos de riesgo que pueden enfrentar los hijos e hijas en un futuro no lejano.

Durante la evolución de la humanidad se pueden identificar cambios en los sistemas de educación parental y cada vez se evidencia mayor involucramiento emocional en las relaciones entre padres e hijos. Este aspecto crucial del proceso de crecimiento se relaciona con los cambios legislativos que dan lugar al reconocimiento del niño como sujeto de derechos, lo cual ha propiciado cambios sociales y culturales que propenden al bienestar integral 
del menor. De allí que los padres y cuidadores reflexionen y adapten sus estilos de crianza procurando favorecer un adecuado desarrollo socioemocional de los niños.

Aunque la crianza y la forma en la que se ejerce existe desde siempre, es posible afirmar que las pautas de crianza son un término acuñado a partir de la década de $1960 . \mathrm{Su}$ primera abanderada fue la psicóloga Diana Baumrind y así lo corroboran Torio, Peña y Rodríguez (2008), quienes consideran que los estudios de la mencionada psicóloga demuestran que la manera en la cual se dan las relaciones de autoridad entre padres e hijos, influye en el proceso de socialización de los infantes. Estos autores mencionan, asimismo, las tres tipologías de padres identificadas por Baumrind: 1) Padres autoritarios: son controladores e inflexibles, exigentes en cuanto a las acciones de sus hijos y poco afectivos. La relación padres e hijos se da de manera jerárquica y el padre ejerce toda la autoridad, desconociendo así las necesidades y derechos del niño. Este tipo de educación puede generar baja autoestima, dependencia y comportamientos agresivos en los niños. 2) Padres democráticos: buscan enseñar a sus hijos normas y valores mediante el razonamiento y la negociación. Suelen dirigir las acciones de sus hijos de manera racional, reflexionan acerca de los derechos y deberes que cada miembro familiar tiene con respecto al otro y hay reciprocidad jerárquica. Este estilo de crianza se caracteriza por la comunicación entre padres e hijos y hace énfasis en la responsabilidad social de las acciones y el desarrollo de la autonomía e independencia en el hijo. Dicho estilo produce, en general, efectos positivos como el desarrollo de competencias sociales, altos índices de autoestima y bienestar psicológico y menor frecuencia de conflictos entre padres e hijos. 3) Padres permisivos: proporcionan gran autonomía al hijo, se comportan de manera flexible y aceptan sus acciones e impulsos. Evitan controlar e imponer autoridad, no marcan límites y son poco exigentes en cuanto a las expectativas de responsabi- lidad de sus hijos. Aparentemente, este tipo de padres forman niños alegres y vitales, pero dependientes, con altos niveles de conductas antisociales y bajo nivel de madurez y éxito personal (Torío, Peña y Rodríguez, 2008).

Se entiende por competencias parentales la adquisición y continua evolución de conocimientos, actitudes y destrezas para conducir el comportamiento parental propio, a través de diversas situaciones de la vida familiar, la crianza y en las distintas dimensiones y necesidades (física, cognitiva, comunicativa, socioemocional) del desarrollo del niño, con la finalidad última de garantizar su bienestar y el ejercicio pleno de sus derechos humanos (Gómez y Muñoz, 2015). Partiendo de dicha afirmación, Gómez y Muñoz (2015) identifican cuatro áreas que hacen parte de las competencias parentales. La primera competencia parental es vincular y se define como la capacidad de promover un estilo de apego seguro y un adecuado desarrollo socioemocional de los niños mediante la sensibilización, el involucramiento y la calidez emocional en la relación padres e hijos. La segunda competencia es formativa, favorece el desarrollo, aprendizaje y socialización de los niños mediante la estimulación del aprendizaje, la incorporación de normas y hábitos, además de la promoción de las relaciones sociales. La tercera competencia es protectora, se dirige al cuidado y protección adecuada de los hijos, mediante la cual se resguardan sus necesidades en cuanto a desarrollo humano, se garantizan sus derechos y se favorece su integridad física, emocional y sexual. Por último, se encuentra la competencia parental reflexiva que es aquella que permite pensar acerca de las influencias y trayectorias de la propia parentalidad, monitorear las prácticas y evaluar el curso del desarrollo del hijo para retroalimentar las otras áreas de competencia parental.

\section{Habilidades sociales}

Dada la importancia de las relaciones sociales en el desarrollo psicoemocional 
del individuo, el estudio de las habilidades sociales ha cobrado relevancia en las últimas décadas. Sin embargo, no existe unanimidad en el concepto, pues algunas características comunes en su definición establecen que son comportamientos aprendidos del contexto de cada individuo, no son un rasgo de la personalidad y están sujetas a las respuestas específicas del individuo ante situaciones concretas. Son muchos los autores y definiciones de habilidades sociales. Así lo ratifica De Miguel (2014) cuando aduce que:

Las habilidades sociales son el conjunto de conductas emitidas por un individuo en un contexto interpersonal, que expresa y recibe opiniones, sentimientos y deseos, que conversa, que defiende y respeta los derechos personales de un modo adecuado, aumentando la probabilidad de problemas en las interacciones sociales. (p. 18).

Diversas investigaciones demuestran que las habilidades sociales inciden en la autoestima, en la adopción de roles, en la autorregulación del comportamiento y en el rendimiento académico, tanto en la infancia como en la adultez (Monjas Casares, 2002; Ovejero Bernal, 1998). Caballo (2007) complementa lo anterior al corroborar que las habilidades sociales son conductas que le permiten al individuo expresar sentimientos, actitudes, deseos, opiniones o derechos de un modo adecuado de acuerdo con la situación.

Por otro lado y de acuerdo con Monjas Casares (2002), Caballo define seis áreas para la evaluación y desarrollo de las habilidades sociales: 1) habilidades básicas de interacción social, asociadas con conductas como sonreír, saludar, presentarse, despedirse, entre otras, y que son necesarias y básicas en el niño para que se relacione con cualquier individuo, sea uno de sus pares o un adulto; 2) habilidades para hacer amigos, necesarias para el inicio, desarrollo y mantenimiento de interacciones sociales positivas con sus pares. Para ello, se requiere del reforzamiento de los otros, iniciaciones sociales, unirse al juego con otros y la cooperación; 3) habilidades conversacio- nales, que permiten al niño iniciar, mantener y finalizar conversaciones con otras personas y comprender habilidades como comenzar y terminar conversaciones tanto de manera individual como grupal; 4) la cuarta habilidad está relacionada con los sentimientos, emociones y opiniones, se relaciona con la asertividad, para lo cual es necesario desarrollar la habilidad de expresar y reconocer las emociones, tanto propias como las ajenas, además de defender los propios derechos sin desconocer los del otro; 5) habilidades de solución de problemas, que corresponden a la capacidad del niño para solucionar de manera adecuada, y por sí mismo, los problemas interpersonales que se le presentan con otros niños; y 6 ) habilidades para relacionarse con los adultos, que comprende la relación con personas de estatus superior, entendiendo que la relación del niño con el adulto es distinta a la que se da con otros niños y abarca las habilidades del niño para relacionarse con el adulto mediante conversación, cortesía, peticiones, refuerzo y solución de problemas.

Para Lacunza y González (2009), el desarrollo de las habilidades sociales se asocia con la evolución, pues consideran que la infancia y la adolescencia son etapas muy favorables para la adquisición y afianzamiento de habilidades. Durante la primera infancia se consideran primordiales las aptitudes del niño para iniciar y mantener el juego, y en la medida que el niño avanza en la adquisición del lenguaje, toman relevancia las habilidades verbales y de interacción con sus pares. Posteriormente, en la etapa preescolar, las habilidades sociales requieren de la interacción con pares, lo que se da principalmente mediante el juego, y así , el niño pasa de un juego solitario al juego grupal. Es entonces cuando la cooperación y la ayuda se observa como las primeras manifestaciones prosociales del individuo. Finalmente, Lacunza y González (2009) destacan lo conductual y cognitivo como ejes principales para la descripción de habilidades sociales. No obstante, es muy importante considerar los contextos situacionales y culturales del individuo. 


\section{Método}

Esta investigación se realizó bajo un enfoque mixto. Se utilizaron técnicas de encuesta y observación. En todo el proceso estadístico se utilizó el software Statistica que posibilitó explicar las relaciones entre las pautas de crianza y las habilidades sociales de hijos únicos. La investigación no fue experimental en tanto que no se modificaron las variables y solo se observaron las características de la muestra, sin ningún tipo de control o manipulación por parte de las investigadoras.

\section{Población y muestra}

La población objeto de estudio la conformó un grupo de 44 familias cuyos hijos acuden al Centro Infantil Castillo de Sueños del municipio de Apartadó, Antioquia (Colombia) con hijos únicos de edades entre los 3 y 5 años. Se tomó una muestra de 36 niños que corresponde al $52 \%$ de la población total de la institución. La escolaridad corresponde a párvulos, prejardín y jardín; asimismo se trabajó con 61 padres y madres de familia para cuya selección se tomó como criterio la voluntad de los padres para participar en la investigación. Estas familias presentaban buena calidad de vida, el $100 \%$ eran profesionales de nivel socioeconómico alto.

\section{Instrumentos}

La Escala de Parentalidad Positiva (Gómez y Muñoz, 2015) es un cuestionario sencillo que responde un adulto responsable de la crianza de un niño. Su objetivo es identificar aquellas competencias parentales que los adultos encuestados utilizan al relacionarse con el niño a su cargo y se agrupa en cuatro áreas: vínculo, formación, protección y reflexión. El cuestionario consta de 54 preguntas y da cuenta de los comportamientos cotidianos de crianza que refleja el despliegue de la competencia parental en estas cuatro áreas. Su respuesta presenta cuatro opciones: casi nunca, a veces, casi siempre y siempre. Este instrumento se sustenta en las siguientes teorías: la de resiliencia humana de Gómez y Kotliarenco (2010), la del apego de Bowlby (1969) y Ainsworth, Blehar, Waters y Wall (1978) y la de ecosistema del desarrollo humano de Bronfenbrenner y Evans (2000).

La escala de habilidades sociales de Lacunza y González (2009) es un cuestionario que aplican los padres y/o cuidadores a niños con edades de 3 a 5 años. Su objetivo es medir los comportamientos observables y da cuenta del desempeño social del menor durante los últimos tres meses. Este cuestionario tiene como base otros instrumentos que miden las habilidades sociales, pero hace una adaptación de acuerdo a las etapas evolutivas del desarrollo en menores preescolares y genera así tres cuestionarios de acuerdo con la edad. Para los menores que tienen 3 y 4 años la escala tiene un cuestionario de 12 ítems, mientras que para aquellos con 5 años de edad, el cuestionario está conformado por 15 ítems. Cada pregunta ofrece tres opciones de respuesta y un valor para cada una de ellas: nunca (N) 1 punto, algunas veces (A/V) 2 puntos y frecuentemente (F) 3 puntos. A mayor puntuación, más alto será el desarrollo de las habilidades sociales del menor.

\section{Resultados}

La población de la presente investigación estuvo conformada por 35 madres y 26 padres de familia con edades entre 24 y 53 años. En relación con la educación, el $90 \%$ eran profesionales de diferentes áreas disciplinares, empleados en diversas instituciones gubernamentales y privadas.

Al comparar el total de las competencias parentales y el género, se encontró la única relación significativa entre estas variables (Chi-cuadrado de Pearson: 6.64707; gl = 2; $p=.03603)$. En la Figura 1 se observa que las competencias parentales óptimas, en ambos géneros, presentan un comportamiento muy similar; sin embargo, se evidencia una gran 
diferencia entre géneros en la zona de monitoreo $^{1}$, en la cual las mujeres presentan un alto porcentaje $(37.37 \%)$ en comparación con los hombres $(22.53 \%)$. En la zona de riesgo, los padres presentan un alto porcentaje $(19.31 \%)$ en comparación con las madres $(2.38 \%)$. Este resultado indica que el género incide en las competencias parentales de los padres.

Por otra parte, no se encontró una relación significativa al comparar el total de las competencias parentales con la edad (Chi-cuadrado: 1.983984; $\mathrm{gl}=4 ; p=.73870)$. Sin embargo, se evidencia una tendencia al aumento en los porcentajes de las competencias en zona óptima y lo contrario en la zona de riesgo, a mayor edad, disminuye la zona de monitoreo entre los padres encuestados.

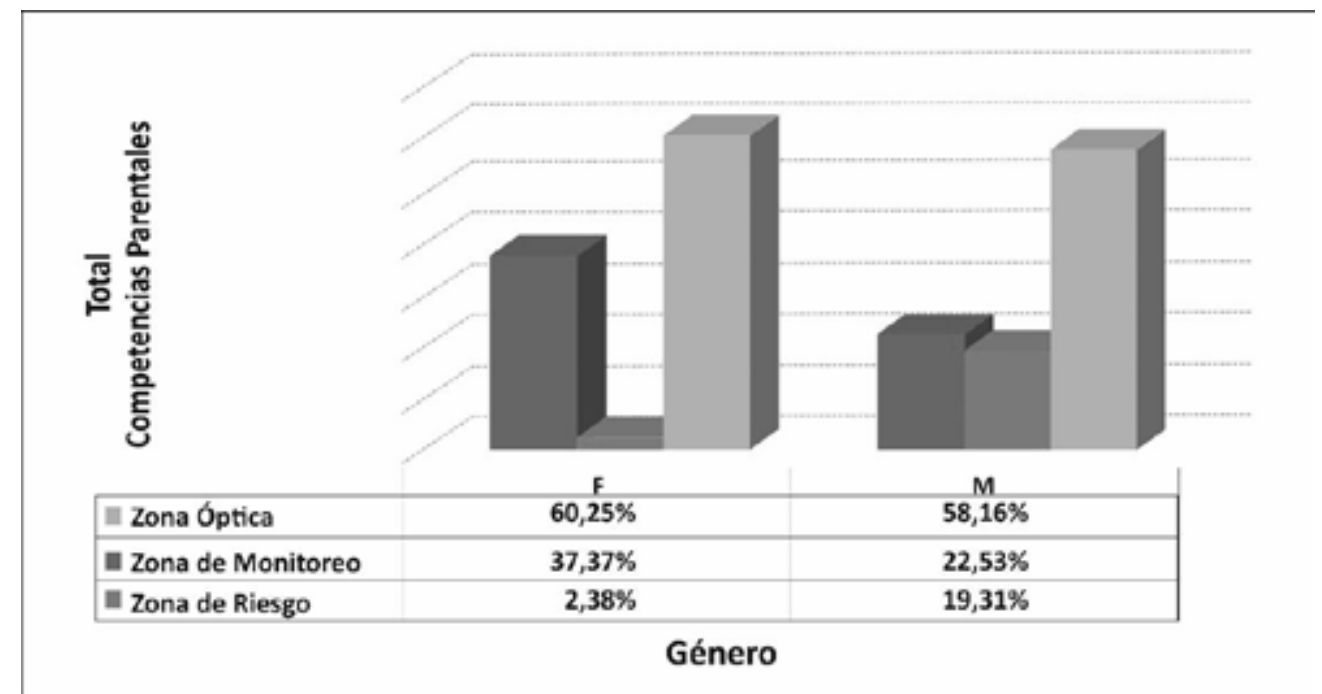

Figura 1. Relación entre las competencias parentales y el género de los padres. Fuente: elaboración propia.

Al analizar la Figura 2, se identifica una relación significativa entre la percepción de las competencias parentales protectoras y el género de los padres encuestados (Chi-cuadrado: $9.519352 ; \mathrm{gl}=2 ; p=.00857)$. Las mujeres obtuvieron puntajes más altos en la zona óptima (37.14 \%) y en la zona de monitoreo $(51.43 \%)$. En tanto los hombres presentaron altos porcentajes en la zona de riesgo $(46.15 \%)$.
En cuanto a las competencias parentales vinculares y el género, las mujeres presentan porcentajes más altos en la zona óptima (51.43 $\%$ ) en comparación con los hombres (38.46 $\%)$, en tanto que los padres presentan mayores porcentajes en la zona de riesgo $(38.46 \%)$ con respecto al de las madres (14.29\%) (Chi-cuadrado: 4.72742; $\mathrm{gl}=2 ; p=.09407)$.

\footnotetext{
1La Escala de Parentalidad Positiva define tres zonas: La primera es la zona de riesgo, donde se ubica a los padres cuyos puntajes están por debajo de la muestra de referencia. La segunda hace alusión a la zona de monitoreo, en la que se ubica a los padres que presentan puntajes con porcentajes entre 30 y $40 \%$ de acuerdo con la muestra de referencia de la tabla. Por último, se define la zona óptima, en la cual se ubica a los padres con puntajes altos.
} 


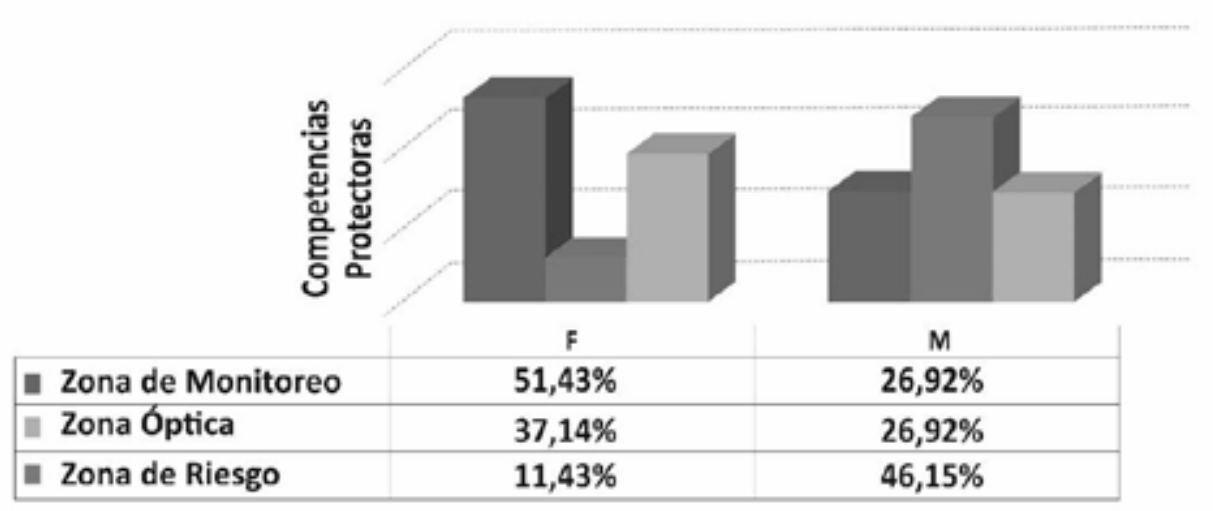

Género

Figura 2. Relación entre las competencias parentales protectoras y el género de los padres. Fuente: elaboración propia.

Al analizar la percepción de competencia parental, se encontró que no existen diferencias significativas entre padres y madres objetos de estudio sobre sí mismos. Por el contrario, en las competencias parentales formativas y reflexivas hubo diferencias dependiendo de género y edad. Se hallaron datos importantes con respecto al comportamiento de las competencias vinculares (Chi-cuadrado: 4.72742; $\mathrm{gl}=2 ; p=.09407)$ y protectoras de acuerdo al género (Chi-cuadrado: 9.519352; gl $=2$; $p=.00857)$.

Así pues, en el análisis de las competencias parentales se identificó que existen diferencias entre mujeres y hombres, lo cual se hace evidente al encontrar que en la zona óptima, ambos géneros se encuentran en porcentajes muy similares: $60.25 \%$ mujeres y $58.16 \%$ hombres. Sin embargo, es interesante que las mujeres $(37.37 \%)$ se encuentran en la zona de monitoreo en mayor medida que los hombres $(22.53 \%)$, mientras que los padres $(19.31 \%)$ presentan índices más altos que las madres $(2.38 \%)$ en la zona de riesgo. Se podría decir que al sobre exigirse con los hijos para desempeñar su rol biológico y social de cuidadoras, las madres se ocupan de organizar la vida cotidiana y ofrecer contextos que generen seguridad al menor, lo cual fortalece sus competencias para protegerlos y proporcionarles bienestar. Sin embargo, al asumir labores fuera del hogar, pueden llegar a ser más flexibles con respecto a algunas condiciones de cuidado y vinculación emocional con sus hijos, lo cual conlleva que sus competencias se encuentren en la zona de monitoreo. En la contraparte, los hombres siguen ejerciendo su rol de proveedores con responsabilidades limitadas en la crianza y pueden llegar a ser evasivos y/o negligentes con respecto a sus deberes de protección y vinculación con sus hijos.

Asimismo, si se toma en consideración que el primer vínculo afectivo se establece con la madre y que el sentimiento maternal proporciona afecto e intimidad a los hijos, mientras que el padre suele asociarse más a la disciplina y la independencia, se podría deducir que es más fácil para las mujeres desarrollar competencias parentales vinculares, las cuales están relacionadas con el adecuado desarrollo emocional del niño en cuestión.

Al comparar el desarrollo de las competencias de acuerdo con la edad, no se evidenciaron diferencias significativas entre los padres y madres que hicieron parte de la investigación. Sin embargo, sí se halló un leve aumento en la frecuencia con la que los padres con mayor edad clasificaban dentro de la zona óptima y una disminución en la zona de monitoreo, tal vez como consecuencia de la madurez emocional, 
la mengua de la impulsividad, mayor estabilidad económica y compromiso, y deseo de tener hijos. Se podría afirmar que, a mayor edad, los padres se adaptan más a la crianza de los hijos, se centran en situaciones y condiciones relevantes y logran así desarrollar de manera más adecuada sus competencias en el rol de padres. Con respecto al comportamiento de la muestra en la categoría de edad de 43 a 54 años, se halló que el porcentaje de padres en zona de riesgo es mayor que en los otros grupos de edad. Se considera que este fenómeno está más relacionado con el género que con la edad, siendo que el $80 \%$ de este grupo lo constituyen hombres, solo $20 \%$ son mujeres.

\section{Habilidades sociales}

En la investigación participaron 36 menores: el $50 \%$ (18) niñas y la otra mitad niños, distribuidos así: 17 niños/niñas de 3 años; 15 infantes entre niñas y niños de 4 años y 4 niños de 4 años. Un detalle significativo es la ausencia de niñas de cinco años. Como se sabe, en el periodo entre los tres y cinco años, de acuerdo a Lacunza, Castro y Contini (2009) se desarrollan las capacidades sociales, lo que le permitirá su incorporación al mundo social de una forma amplia y segura. En concordancia, Monjas (2002) ratifica que, durante los primeros años de vida, la figura de apego representada en la familia es determinante para el comportamiento del infante. Esto se debe a que la familia es el contexto principal donde vive y se desarrolla el niño, le proporciona las oportunidades sociales, seguridad, afecto, nutrición, educación y posibilita la incorporación a otros contextos, fortaleciendo así sus habilidades para interactuar socialmente.

Frente a los resultados, al comparar la habilidad para relacionarse con adultos y el género se identificó que el $88.89 \%$ de las niñas demostraron mayor habilidad en esta área, en comparación con el $61.11 \%$ de los niños. Con el análisis estadístico se encontró que edad y género no inciden sustancialmente en el desarrollo de las habilidades sociales en los niños y niñas objeto de investigación (Chi-cuadrado: 3.703704; $\mathrm{gl}=1 ; p=.05429$ ).

Con respecto a las habilidades para relacionarse con los adultos y la edad, se encontró una relación significativa (Chi-cuadrado: 14.70850; $\mathrm{gl}=2 ; p=.00064)$. Además, se evidenció que la población de 4 años presenta el porcentaje más alto de desarrollo de esta habilidad ( 93.33 \%) y la población de 5 años presentó un $100 \%$ en puntuación media (Figura 3). Es importante resaltar que el $60 \%$ de la población de 4 años de la presente investigación estuvo integrada por niñas, mientras que el $100 \%$ de la población de 5 años eran niños.

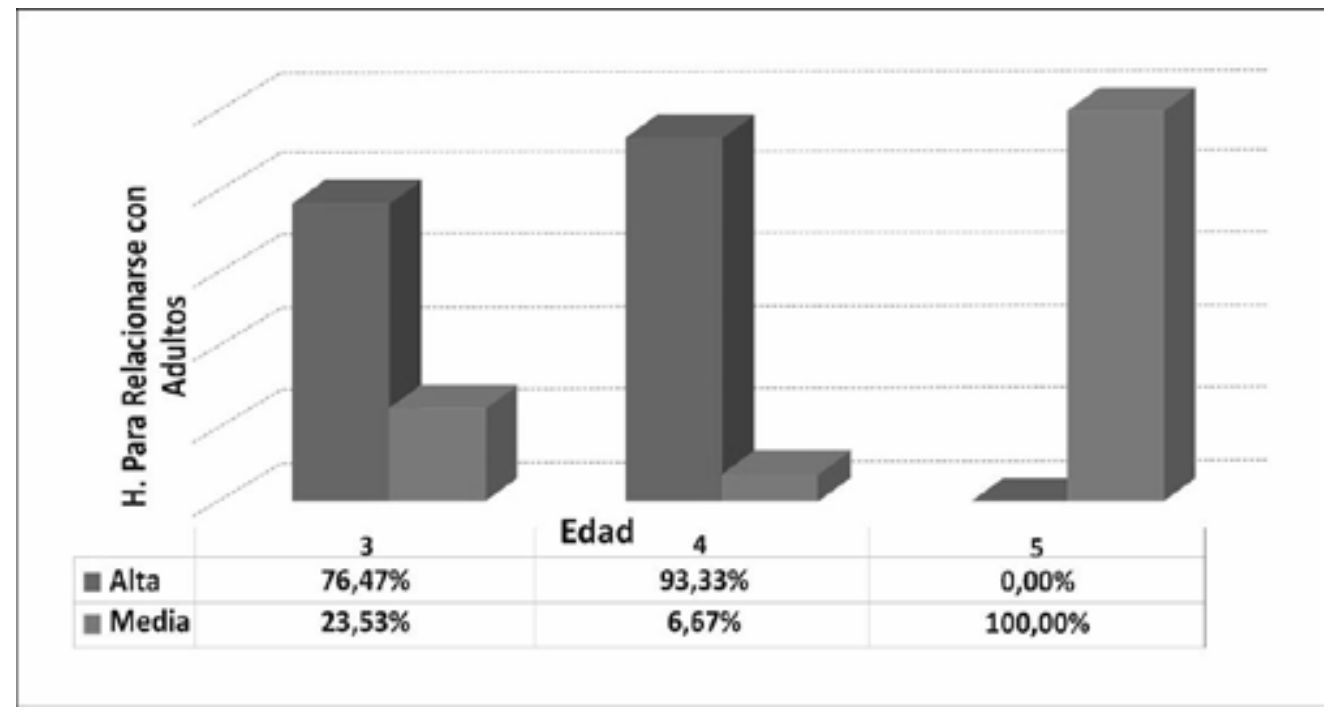

Figura 3. Relación entre las habilidades para relacionarse con adultos y la edad Fuente: elaboración propia. 
Los datos estadísticos evidencian una relación significativa entre la edad y la habilidad para resolver y afrontar problemas (Chi-cuadrado: 6.000257; gl = 2; $p=.04978$ ). El 86.67 $\%$ de la población de 4 años obtuvo un puntaje alto en esta habilidad, mientras que en el grupo que tenía 5 años, el $75 \%$ presentó habilidad media, lo cual evidencia menor desarrollo de esta habilidad en comparación con los niños de 3 y 4 años (Figura 4).

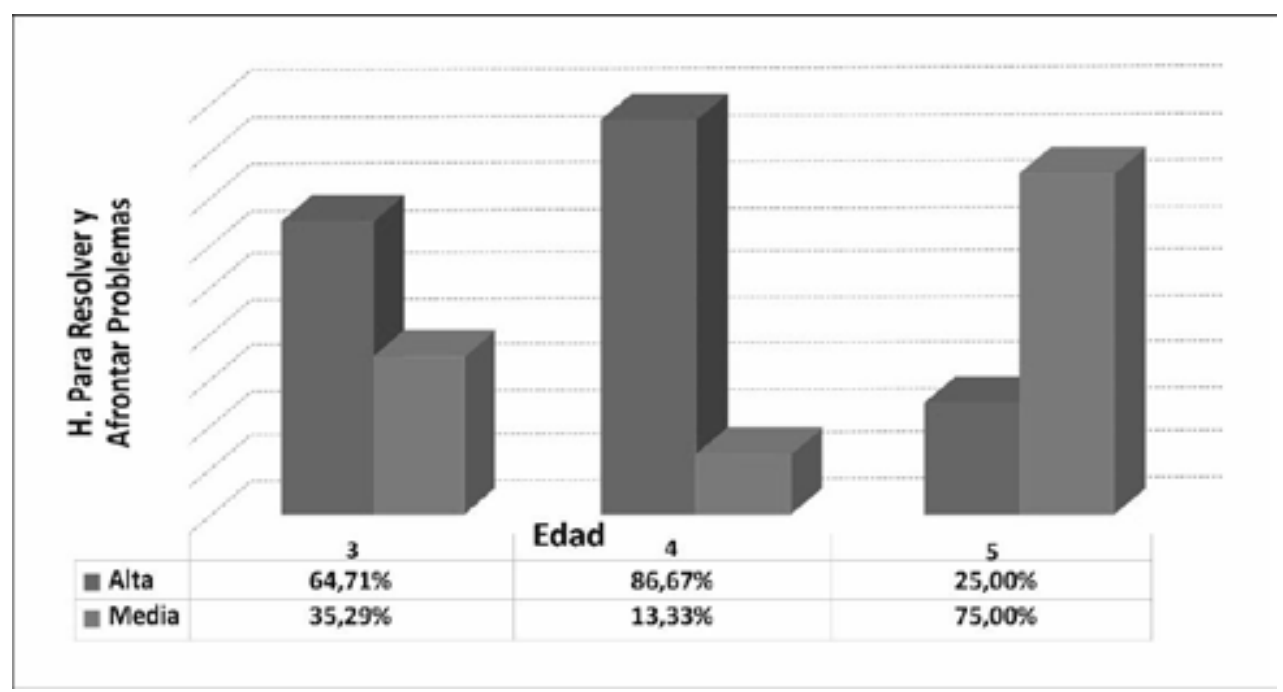

Figura 4. Relación entre las habilidades para afrontar y resolver problemas y la edad Fuente: elaboración propia.

Al comparar las habilidades para expresar emociones y sentimientos en relación con la edad, se evidenció una relación significativa (Chi-cuadrado: 24.47033; $\mathrm{gl}=4 ; p=.00006$ ), la edad incide ostensiblemente a la hora de expresar emociones y sentimientos. Además, se observó que la población de 4 años presentó el porcentaje más alto de desarrollo de esta habilidad $(86.67 \%)$, la población de 5 años obtuvo $50 \%$ una puntuación media y el resto con puntuación baja. Se demuestra por tanto que, a mayor edad, menor habilidad para expresar emociones y sentimientos, al comparar con la población de 3 y 4 años. De acuerdo con el análisis de los datos estadísticos, se encuentra mayor habilidad en las niñas en la mayoría de las áreas (habilidades básicas, habilidades para expresar emociones, habilidades para resolver problemas, habilidades para relacionarse con los adultos). Sin embargo, en las habilidades para hacer amigos se evidencia un mayor desarrollo en los niños y ante esto, se podría tomar en consideración el rol que culturalmente se la atribuye a la mujer desde pequeña en la asignación de responsabilidades por parte de los padres, puesto que tradicionalmente se protege más a las niñas, pero a partir de los tres años se les empieza a exigir colaborar con las madres o cuidadores en labores simples del hogar, lo cual las obliga a resolver pequeños problemas cotidianos. Esto podría ser un factor determinante para el desarrollo de sus habilidades.

En cuanto a la diferencia que se da con respecto a las habilidades para hacer amigos, se pueden formular varias interpretaciones: en estas edades, los menores, en general, tienden a la relacionarse en mayor medida con su mismo género; a medida que van creciendo, las niñas se van volviendo menos abiertas con los varones y privilegian la compañía del mismo género. Si bien la investigación también muestra que se privilegian las relaciones entre niñas, esas relaciones no están exentas de una marcada rivalidad.

La experiencia muestra que se establecen relaciones muy intensas desde el afecto en las niñas, pero muy cargadas también de un aspecto 
negativo que anida en una díada amor-odio muy significativo. Esta rivalidad podría estar asociada con la explicación que la escuela psicoanalítica da a ciertos comportamientos femeninos en esta edad, y tiene que ver con el complejo de Electra, por el cual la niña se percibe incompleta (ausencia de pene) y percibe que su madre comparte esta condición. Esto puede marcar el inicio de una rivalidad compleja entre madre e hija por el amor del padre y por su atención, que puede afectar la capacidad de la niña para iniciar y mantener relaciones de amistad entre sus iguales, y la niña trata de resolver buscando complemento en el amor del padre. Una interpretación, desde lo filogenético, podría sugerir que biológicamente la niña se repliega en relaciones más íntimas porque en la adultez va a requerir cuidar de la familia y esto la va a sustraer parcialmente de lo social, lo que ha sido un rol histórico en la cultura occidental. En dicho contexto, el varón se sobreexige en las relaciones sociales porque necesita esa habilidad para triunfar en el mundo externo y llegar a ser un buen proveedor para la familia.

Otro dato interesante recolectado en esta investigación se relaciona con la disminución de todas las habilidades en la población de 5 años. No obstante, cabe acotar que la población de 5 años solo representa el $11.11 \%$ del total de la población encuestada y que, además, el 100 $\%$ de los participantes con edad de 5 años eran de género masculino. Esta preponderancia se podría considerar como una variable fantasma que justifique la disminución en la habilidad a los 5 años, pues no tiene sentido, desde el sustento teórico, que los niños y niñas lograran el pico más alto en la habilidad a los 4 años y lo perdieran drásticamente a los 5 años. Se considera que es más un asunto de género que de edad, lo cual deja abiertas muchas preguntas para futuras investigaciones.

\section{Competencias parentales y habilidades sociales}

Se encontró una relación significativa entre las competencias parentales vinculares y las habilidades sociales totales (Chi-cuadrado: 9.349579; $\mathrm{gl}=2 ; p=.00933)$. El análisis indica que los padres con competencias vinculares óptimas tienen hijos con habilidades sociales medias, mientras que los niños con habilidades sociales altas tienen padres cuyos porcentajes son similares en las tres áreas de evaluación de las competencias vinculares.

Los resultados demostraron una fuerte dependencia entre las competencias parentales vinculares y las habilidades de los niños para relacionarse con adultos (Chi-cuadrado: 6.444201; gl $=2 ; p=.03987)$. Los padres con competencias vinculares óptimas tienen hijos con habilidades para relacionarse con los adultos en un puntaje medio, mientras que aquellos niños con mayor desarrollo de esta habilidad tienen padres que presentan porcentajes similares en las tres zonas de evaluación.

Con respecto a la relación de las competencias parentales vinculares con las habilidades para hacer amigos no se presentó una correlación fuerte (Chi-cuadrado: 5.480318; $\mathrm{gl}=2 ; p$ $=.06456)$. Se nota que los niños con altas habilidades para hacer amigos tienen padres con similares puntajes en la zona óptima (39.02 $\%$ ) y de monitoreo (39.02\%), mientras que los padres de los niños cuyas habilidades para hacer amigos clasificaron en el rango medio, presentan un alto porcentaje de competencias vinculares óptimas $(60 \%)$ y un incremento de las competencias vinculares en zona de riesgo (30\%).

No se halló una relación significativa entre las competencias parentales vinculares y las habilidades básicas, es decir que dichas variables no se influyen entre sí (Chi-cuadrado: 4.321177; $\mathrm{gl}=2 ; p=.11526)$. El análisis de estas variables muestra que los padres con competencias vinculares óptimas se correlacionan con hijos que presentan habilidades básicas con puntuación media. Entre tanto, los padres de los niños con habilidades básicas altas presentan porcentajes de puntuación similares en las zonas de monitoreo $(37.21 \%)$ y la zona óptima (39.53\%) de evaluación de las competencias vinculares. 
Del mismo modo, no se observa relación significativa entre las competencias parentales protectoras y las habilidades de los niños para relacionarse con adultos (Chi-cuadrado: 8.567535; $\mathrm{gl}=2 ; p=.65157)$. Se puede evidenciar que los niños obtuvieron mayor puntaje en el desarrollo de esta habilidad, lo cual se relaciona con padres con competencias parentales protectoras en zona de monitoreo (44.44\%), por encima de los padres con competencias en zona óptima (31.11\%). Pero, los padres de los menores con estas habilidades en puntuación media obtuvieron porcentajes muy similares en las tres zonas de evaluación de las competencias protectoras, siendo mayor el porcentaje de padres en zona óptima (37.50 \%).

Los resultados obtenidos al correlacionar las competencias parentales protectoras y las habilidades para resolver y afrontar problemas entre padres e hijos, no muestran una influencia ostensible (Chi-cuadrado: 4.523970; $\mathrm{gl}=2 ; p$ $=.10414)$. Sin embargo, es importante resaltar que los niños con puntajes altos en las habilidades para resolver y afrontar problemas tienen padres que se encuentran en mayor proporción en la zona de monitoreo (48.84\%), presentan porcentajes iguales en la zona de riesgo (25.58 \%) y la zona óptima (25.58 \%). El análisis de las competencias protectoras y su relación con las habilidades para expresar emociones y sentimientos muestra que no existe una relación significativa entre estas variables (Chi-cuadrado: 5.055976; $\mathrm{gl}=4 ; p=.28160$ ).

Fue posible identificar datos importantes acerca del comportamiento de las competencias parentales protectoras y el desarrollo de las habilidades para expresar emociones y sentimientos. Al igual que en los casos anteriores, se halló que los niños que más habían desarrollado habilidades para expresar emociones provienen de padres con un alto porcentaje en la zona de monitoreo de competencias protectoras (48.48 $\%)$. Entre tanto, los niños que presentaron puntajes medios provienen de padres que se encuentran en las tres zonas de evaluación de las competencias, en porcentajes muy similares. Como caso excepcional, se observó que en la habilidad para expresar emociones y sentimientos había niños con desempeño bajo cuyos padres obtuvieron un alto porcentaje en zona de riesgo en cuanto a competencias protectoras $(66.67 \%)$, mientras que el resto $(33.33 \%)$ se ubicó en la zona de monitoreo.

No se encontró una afectación sustancial de las habilidades de los niños para relacionarse con adultos, en contraste con las competencias parentales protectoras (Chi-cuadrado: 8.567535; $\mathrm{gl}=2 ; p=.65157)$. Como consecuencia, se puede observar que los niños que mostraron mayor puntaje en el desarrollo de esta habilidad se correlacionan con padres cuyas competencias parentales protectoras se ubican en la zona de monitoreo (44.44 \%), por encima de los padres con competencias en zona óptima $(31.11 \%)$. Entre tanto, los padres de los menores con estas habilidades en puntuación media, presentan porcentajes muy similares en las tres zonas de evaluación de las competencias protectoras, siendo mayor el porcentaje de padres en zona óptima (37.50\%).

La relación entre las competencias parentales protectoras y las habilidades de los menores para resolver y afrontar problemas no es significativa (Chi-cuadrado: 4.523970; gl $=2 ; p=.10414)$. Sin embargo, es importante conocer que los padres de los niños que obtuvieron puntajes altos en las habilidades para resolver y afrontar problemas, se ubicaron en mayor medida en la zona de monitoreo (48.84 $\%)$, y presentaron porcentajes iguales en la zona de riesgo $(25.58 \%)$ y la zona óptima $(25.58 \%)$.

En el análisis de las competencias protectoras y su correlación con habilidades de los niños para expresar emociones y sentimientos, se ratificó que no existe una relación significativa (Chi-cuadrado: 5.055976; $\mathrm{gl}=4 ; p=$ .28160). Allí emergen datos importantes al respecto. Al igual que en los casos anteriores, se observó que los niños con mayor habilidad para expresar emociones provienen de padres situados en la zona de monitoreo (48.48\%) con respecto a las competencias protectoras. Los menores que obtuvieron una evaluación 
media provienen de padres que se encuentran en las tres zonas de evaluación de las competencias y en porcentajes muy similares. Como caso único, se observó que para la habilidad de expresar emociones y sentimientos había niños con un desempeño bajo provenientes de padres con un alto porcentaje en zona de riesgo (66.67 $\%$ ) de sus competencias protectoras, el resto $(33.33 \%)$ se ubicó en la zona de monitoreo.

En cuanto a la relación de las competencias parentales protectoras con las habilidades conversacionales no se encontró una influencia significativa (Chi-cuadrado: 4.523970; $\mathrm{gl}=2$; $p=.10414)$. No obstante, es importante considerar la continuidad en el comportamiento de la relación entre estas dos variables, puesto que se puede observar que los niños con puntajes altos en las habilidades conversacionales se asocian a padres cuya competencia protectora se encuentran en mayor porcentaje en la zona de monitoreo (45.45\%).

No se encontró influencia de las competencias parentales protectoras en las habilidades para hacer amigos (Chi-cuadrado: .9194634; $\mathrm{gl}=2 ; p=.63145$ ). Se observó que los niños con más alto puntaje en el desarrollo de esta habilidad contaban con padres cuyas competencias parentales protectoras se ubicaban en la zona de monitoreo (41.46\%), mientras que los menores que obtuvieron una puntuación media para estas habilidades se asociaban a padres con porcentajes iguales en la zona óptima y la zona de monitoreo (40\%).

En la relación de las competencias parentales protectoras con las habilidades básicas se identificó que los niños con mayor desarrollo de esta habilidad presentaban vínculo con padres, en su mayoría, situados en la zona de monitoreo de sus competencias protectoras (48.84 $\%)$. Los niños que presentaron puntuación media en esta habilidad provenían de padres con iguales porcentajes tanto en la zona óptima como en la de riesgo ( $38.89 \%$ ) y, a su vez, una menor representación en la zona de monitoreo $(22.22 \%)$. El análisis estadístico de esta relación no resultó ser significativa (Chi-cuadrado: 4.043221; $\mathrm{gl}=2 ; p=.13244)$.
Se encontraron características particulares con respecto a la relación estadística entre las competencias parentales y las habilidades sociales de los menores en la asociación de las competencias vinculares y las competencias protectoras con las habilidades sociales de los niños.

Al momento de asociar las competencias vinculares con las habilidades sociales se hallaron datos interesantes acerca de su influencia en las habilidades sociales de los menores Se encontró que los niños que presentaron habilidades sociales altas provenían de padres con porcentajes muy similares en las tres áreas de evaluación de las competencias parentales (zona óptima, de monitoreo y de riesgo). En cambio, los niños con habilidades de puntajes medios provenían de padres con competencias vinculares óptimas en un alto porcentaje.

Al evaluar la correlación de las competencias protectoras con las seis áreas de las habilidades sociales, los niños con habilidades altas se asocian a padres con un alto porcentaje en la zona de monitoreo. Los menores que presentaron habilidades sociales medias se vinculan a padres con porcentajes muy similares en las tres zonas de evaluación de las competencias parentales.

Otro hallazgo se relaciona con la habilidad para expresar emociones y sentimientos: la única habilidad en los niños presentó un puntaje bajo y se asocia a padres con un alto porcentaje de competencias protectoras en zona de riesgo $(66.67 \%)$; el resto $(33.33 \%)$ se ubica en zona de monitoreo.

Los hallazgos de esta investigación no coinciden con lo que en teoría se podría esperar, ya que no tiene sentido que niños que tienen habilidades sociales medias tengan padres con competencias parentales óptimas, mientras que niños con habilidades sociales altas tengan padres que se pueden ubicar en cualquiera de los tres rangos de competencia. El otro hallazgo inesperado es que los niños que presentaron habilidades sociales altas en las seis áreas, se asocian a padres con competencias parentales 
protectoras en zona de monitoreo.

\section{Discusión}

Durante el desarrollo de la investigación fue posible determinar que no existe una correlación significativa entre el desarrollo de competencias parentales de los padres con su género o edad. Frente al género, se observaron algunas diferencias en los resultados de la evaluación de las competencias protectoras y vinculares. Mediante el análisis de las competencias protectoras se estableció que las madres se ubicaban principalmente en zona de monitoreo, mientras que los padres tuvieron mayores puntajes en la zona de riesgo. Al evaluar las competencias vinculares con respecto al género, los hallazgos indicaron que las mujeres presentan altos porcentajes en zona óptima, seguido por la zona de monitoreo, entre tanto los hombres presentan porcentajes muy similares entre los puntajes de la zona óptima y la zona de riesgo. Estos datos permiten deducir que las mujeres desarrollan mayores competencias que los hombres para la crianza de los hijos.

Aunque los resultados no arrojan diferencias significativas en el desarrollo total de las habilidades sociales en menores que conciernan a su género y edad, se evidencia que, en las habilidades de relacionarse con los adultos, las niñas presentan puntajes más altos que los niños, mientras que, en la habilidad para hacer amigos, los niños muestran mayor destreza.

Por otra parte, al comparar la edad con la habilidad para relacionarse con los adultos, el puntaje más bajo de desarrollo en la habilidad para resolver y afrontar problemas y expresar emociones y sentimientos, se encontró en la población de 5 años, mientras que los puntajes más altos se encontraron en la población de 4 años. Es importante resaltar que, en esta investigación, el $60 \%$ de la población de 4 años en la investigación estuvo integrado por niñas, mientras que el $100 \%$ de la población de los 5 años eran niños. Esta preponderancia en uno y otro grupo puede haberse convertido en una variable interviniente que justifique las dife- rencias halladas, especialmente en los casos en los que no coinciden con la experiencia clínica documentada en informes científicos de psicología.

Asimismo, se identificó una relación entre algunas competencias parentales y las habilidades sociales de los menores. Aun cuando no se halló correlación significativa entre las competencias formativas y reflexivas de los adultos y las habilidades sociales de los niños, al evaluar las habilidades sociales y las competencias vinculares, se estableció que los niños con habilidades medias tienen padres con puntajes de competencias en zona óptima. Al mismo tiempo, los niños con habilidades altas provienen de padres con valores muy similares en las tres zonas de evaluación de las competencias vinculares.

Además, se encontró que al vincular las competencias protectoras de los adultos con el desarrollo de las habilidades sociales en los menores, se determinó que los niños con habilidades sociales altas se asocian a padres con competencias protectoras que clasifican, principalmente, en la zona de monitoreo. Estos hallazgos generan ciertos interrogantes al no coincidir con los resultados proyectados, pues se esperaría que los niños con habilidades sociales altas proviniesen de padres con competencias protectoras óptimas.

Las herramientas aplicadas tanto a padres como a hijos no resultan suficientes para determinar si realmente existe una incidencia preponderante de las competencias parentales en el desarrollo de habilidades sociales de los niños. Sin embargo, ofrecen una aproximación a las competencias parentales positivas, las cuales están relacionadas con pautas de crianza adecuadas y además permiten analizar algunas de las conductas sociales de los niños objeto de estudio.

Asimismo, los datos de la investigación aportan información importante acerca de la percepción que tienen los padres con respecto a sus competencias parentales. Al tratar las habilidades sociales de los niños, se observan puntajes altos, pero al evaluar las competencias 
de los padres, se encuentran valores correspondientes a las zonas de monitoreo y de riesgo, principalmente en las competencias protectoras y vinculares. Sin embargo, es importante mencionar que la investigación se llevó a cabo en el marco de condiciones que pueden haber interferido en los resultados. Una de ellas es que la evaluación de las competencias parentales se realizó con un cuestionario autoadministrado, respondido desde la subjetividad de cada padre, que pudo conllevar a un sesgo en la información recolectada debido a que, al realizar el análisis de la información, algunos patrones de comportamiento no coincidían con lo observado previamente en la población de la muestra. Se considera por tanto que la investigación se vio limitada al aplicarse a población adulta con un nivel socioeconómico alto $\mathrm{y}$-mayoritariamente-con formación profesional, por lo que es posible plantear que, debido a sus ocupaciones, los padres dedican un tiempo limitado a la crianza de sus hijos y que, en muchas ocasiones, esta queda a la merced de los cuidadores, quienes finalmente terminan impartiendo los patrones de crianza en los menores. Así pues, se podría pensar que aplicar dichos instrumentos en otros contextos socioeconómicos y culturales podría arrojar datos más significativos sobre la relación entre las competencias en la crianza y el desarrollo de las habilidades sociales de menores en otro ámbito.

Finalmente, se concluye que no hay criterios que indiquen una relación significativa entre las competencias parentales positivas y el desarrollo de las habilidades sociales de los padres e hijos únicos con edades entre 3 y 5 años del Centro Infantil Castillo de Sueños. Además, se encontró que los padres presentan mayor desarrollo de las competencias vinculares, lo cual permite generar un tipo de apego seguro y un adecuado desarrollo socioemocional del menor. Asimismo, los padres parecen tener menos desarrolladas las competencias protectoras dirigidas a cuidar y proteger a sus hijos. Por último, se afirma que la población investigada posee un buen promedio de habilidades de interacción social. Sin embargo, para Gómez y Muñoz (2015) quedan desafíos en el desarrollo del instrumento, aunque se evidencia la validez de la escala de parentalidad positiva (E2P) como mecanismo confiable para analizar las relaciones familiares y su desarrollo en las habilidades sociales tanto de infantes como adolescentes, por lo que queda pendiente que se aplique en diversos y representativos grupos de la región de Urabá, teniendo en cuenta factores como "la curva de ROC, análisis IRT o de respuesta al ítem, estudio de validez predictiva, y otros" (p. 30).

Por otro lado, tratando de incentivar el debate a los resultados, Lacunza, Solano y Contini (2009) expresan que a las habilidades sociales las influyen las características del entorno, donde las malas condiciones de vida pueden generar impacto negativo en el desarrollo de las habilidades sociales. Esta afirmación cobra sentido si se tiene en cuenta que la pobreza y las condiciones precarias de vida pueden generar incertidumbre en el futuro, aumenta los estados emocionales negativos, depresión, diversas enfermedades, circunstancias que pueden afectar el desarrollo infantil como las capacidades cognitivas, sociales $\mathrm{y}$ emocionales.

En la investigación, la población objeto presenta aceptables condiciones de vida, los padres tienen buen status social, profesionales y con actividades laborales seguras, garantizando así calidad de vida a la familia y obviamente a los infantes. Lo paradójico es que los resultados demuestran que muchos padres presentar competencias parentales vinculares y protectoras en zona de monitoreo y riesgo, mientras sus hijos presentan buen promedio de habilidades de interacción social adecuadas según la edad.

Quedan preguntas para futuras investigaciones: ¿por qué los niños con habilidades sociales altas se correlacionaron con padres cuyas competencias parentales protectoras se ubicaron en la zona de monitoreo, cuando se esperaría que fueran hijos de padres con competencias óptimas? ¿Depende el desarrollo 
de las habilidades sociales de los hijos únicos en mayor medida de otros factores que no están asociados a las competencias de sus padres?

\section{Referencias bibliográficas}

Ainsworth, M., Blehar, M., Waters, E. y Wall, S. (1978). Patterns of Attachment: A Psychological Study of the Strange Situation. Hillsdale, NJ: Erlbaum.

Barudy, J. y Dantagnan, M. (2005). Buenos tratos a la infancia: parentalidad, apego y resiliencia. Barcelona: Gedisa.

Bowlby, J. (1969). Attachment and loss: Vol.1. Attachment. New York: Basic Books

Bronfenbrenne, U. y Evans, G.W. (2000). Developmental science in the 21st century: Emerging theoretical models, research design and empirical findings. Social Development, 9, 115-125.

Caballo, V. E. (2007). Manual de evaluación y entrenamiento de las habilidades sociales $\left(7^{\circ}\right.$ ed.). Madrid: Siglo XXI.

De Miguel, P. (2014). Enseñanza de habilidades de interacción social en niños con riesgo de exclusión. Revista de Psicología Clínica con Niños y Adolescentes, 1(1), 17-26. https://goo. $\mathrm{gl} / 55 \mathrm{mxLD}$

Gómez, M. E. y Muñoz, M. M. (2015). Escala de Parentalidad Positiva e2p: manual. ( $2^{\circ}$ ed.). Santiago de Chile: Fundación Ideas para la Infancia.

Gómez, E. y Kotliarenco, M. A. (2010). Resiliencia familiar: un enfoque de investigación e

intervención con familias multiproblemáticas. Revista de Psicología, 19(2), 103-131. https:// core.ac.uk/download/pdf/46532445.pdf

Izzedin, R. y Pachajoa, A. (2009). Pautas, prácticas y creencias acerca de crianza... ayer y hoy. Liberabit, 15(2), 109-115. https://goo.gl/b1hmLN

Lacunza, A. B., Castro S. A. y Contini, N. (2009). Habilidades sociales preescolares: una escala para niños de contextos de pobreza. Revista de Psicología, 27(1), 4-28. https://www.redalyc. org/pdf/3378/337829512001.pdf
Lacunza, A. B. y González, N. C. (2009). Las habilidades sociales en niños preescolares en contextos de pobreza. Revista Oficial de la Facultad de Psicología de la Universidad Católica del Uruguay Dámaso a Larrañaga, (3), 1-14.

Monjas Casares, M. (2002). Programa de enseñanza de habilidades de interacción social (PEHIS) para niños y niñas en edad escolar. Madrid: CEPE, S.L.

Muñoz, J. M. (2009). La importancia de la socialización en la educación actual. Revista Digital Innovación Educativa, (14), 1-9.

Muñoz Silva, A. (2005). La familia como contexto de desarrollo infantil. Dimensiones de análisis relevantes para la intervención educativa $\mathrm{y}$ social Portularia, 5 (2), 147-163.

Ovejero Bernal, A. (1998). Las habilidades sociales y su entrenamiento en el ámbito escolar. En F. Gil y J. León (Comps.) Habilidades sociales. Teoría, investigación e intervención (pp. 169-185). Madrid: Síntesis Psicológica.

Rodrigo López, M. J., Martín Quintana, J. C., Cabrera Casimiro, E. y Máiquez Chaves, M.L. (2009). Las Competencias Parentales en Contextos de Riesgo. Psicosocial Psychosocial Intervention, 18, (2). pp. 113-120. https://www. redalyc.org/pdf/1798/179814021003.pdf

Social Trends Institute. (2014). World family map 2014: mapa de los cambios en la familia y consecuencias en el bienestar infantil. Bethesda, MD: Child Trends. http://www.socialtrendsinstitute. org/upload/2014_WorldFamilyMap_SocialTrendsInstitute_spanish.pdf.

Vargas Rubilar, J., Lemos, V. y Richard, M. C, (2017). Programa de Fortalecimiento parental en contextos de vulnerabilidad social: una propuesta desde el ámbito escolar. Interdisciplinaria, 34(1), pp. 157-172. https://www.redalyc. org/articulo.oa? $\mathrm{id}=18052925010$.

Torío, S., Peña, J. V. y Rodríguez, M. C. (2008). Estilos educativos parentales: revisión bibliográfica y reformulación teórica. Teoría de $L a$ Educación, 20, 151-178. https://goo.gl/a3ejQj 